\title{
Child psychiatry in a new medical school in Hong Kong
}

\author{
The way we teach medical students
}

\author{
C. K. Wong, Senior Lecturer and Consultant Child Psychiatrist, Department of \\ Psychiatry, The Chinese University of Hong Kong, Prince of Wales Hospital, Shatin, \\ NT, Hong Kong
}

There are two medical schools in Hong Kong, that of the University of Hong Kong and that of the Chinese University of Hong Kong. The former has a history of more than 100 years whereas the latter admitted its first batch of students only in 1981. Both use English as the teaching medium and both are recognised by the GMC. I received my undergraduate medical education in the former but have been teaching in the latter for seven years.

Traditionally, until the new medical school came into existence, psychiatry was an unpopular specialty in Hong Kong. But it seems that we are now witnessing a change for the better. For example in 1987 out of a total of 52 of our first batch of graduates, 18 took up psychiatry and this included the two top students. Although by July 1988 only ten (including the two top students) remained in psychiatry, this still gives an exceptionally high percentage of career choice in psychiatry. Moreover, even those who finally switched over to another specialty still expressed the feeling that they took up psychiatry in the first place because they knew they could learn something useful and important for their career, irrespective of the ultimate specialty they decided to choose.

There are a few important factors for this favourable attitude towards psychiatry among our medical students. While I shall concentrate on the contribution of child psychiatry I shall also briefly mention the other factors so as to complete the picture. First, a short history means in general a more open attitude and a readiness to entertain new ideas among members of the medical faculty. It also means we do not have to wrestle with long established unfavourable or even hostile traditions. Secondly, the members of the faculty are from all over the world and this further promotes a sense of openness and a willingness to try new approaches. Thirdly, from the inception of the medical school psychiatry has established itself as a specialty equal in status to other major specialties. The medical curriculum stretches over five years, the first two being pre-clinical and the last three clinical. Psychiatry is taught in the second clinical year and it occupies a ten-week full time clerkship, similar to the other three modules covered: paediatrics, community medicine, and obstetrics \& gynaecology.

Fourthly, the emphasis on the holistic approach in the practice of medicine appears to be relevant. This is not only reflected in various forms of integrated teaching but also in the professional examination. Other than examinations at the end of each of the modules, students have to take Part I of the Final Professional Examination at the end of the second clinical year. The notable feature of this examination is its integrated nature. Other than two essay papers which equally cover the four specialties, each student has to take a clinical examination on a long case. The four departments provide an equal number of patients and each student is given one of them at random. Within the alloted time of one hour the student is expected to cover all relevant areas of the case, not merely the "illness' or "disease'. He is examined for 30 minutes by a panel of four examiners, one from each of the four departments. Usually the examiner of the department which provides the particular patient first spends about 15 minutes to cover the immediately relevant aspects of the case. The other three examiners then ask questions on collateral areas. After conducting this form of examination for a few years, most of us agree that our students are showing encouraging evidence of good divergent thinking in their clinical performance.

Finally, the philosophy of teaching in the Department of Psychiatry itself; although we do give reasonable cover of the major psychiatric topics, we strongly emphasise that our aim is not to teach psychiatry as such but to help our students appreciate the psychiatric aspects of the practice of medicine, irrespective of specialty. We believe that the most powerful and appropriate way to promote psychiatry is paradoxically not to aim to turn students into psychiatrists but to let them appreciate the contributions of psychiatry to medicine in general. In other words, not only do we not feel bad at all if they finally decide on medicine, paediatrics, surgery, etc. but we are actually pleased if we have contributed in 
making them good physicians, paediatricians or surgeons. We convey this philosophy to our students explicitly and repeatedly.

Child psychiatry makes its contribution in this scenario. Unfortunately, we have only one child unit, with only two doctors, namely myself and a trainee at the registrar level. Since the trainee rotates every six months I do not as a rule assign any teaching task to him. The number of students is, in contrast and without exaggeration, overwhelming. We have currently 100 students in the second clinical year but the number will reach the maximum of 140 in two years' time. This means 25 students in one module (with the number increasing to 35). Indeed in Hong Kong shortage of man-power is a way of life. The way we cope may be of interest here. Cost-effectiveness is obviously vital.

The quantity of teaching in child psychiatry roughly amounts to $25 \%$ of all teaching in psychiatry. In the Psychiatry Module the first week is for orientation and the last for revision and examination. For the eight weeks in between students are rotated into two big groups which change over at the middle of the eight weeks. One group does adult psychiatry in the same teaching hospital. The other does child psychiatry with me half of the time and mental hospital psychiatry for the rest of the time.

The modalities of teaching during the Psychiatry Module include: the clinical new case session, the child case conference, and the lecture series.

I run two new case sessions per week. Students are assigned in pairs to a new child patient. They are required to pay the family a home visit a few days before the new case session itself. On the day of the clinic, the student pair interview the family (or whoever finally attends) for about an hour. The other ten students watch over the closed circuit TV system while the interview is being videotaped. One of the observer students is given the responsibility of making a log of the videotape; without this log it is difficult to locate required excerpts on the tape afterwards. I try to be with the observer students, discussing with them issues like the doctor-patient relationship and interviewing techniques. At the end of the hour the family takes a 30-minute break while the two students present their case to the other students and myself. Usually this is followed by a short discussion period. Then comes my turn to see the family for diagnostic and therapeutic purposes. Again the students continue to observe over the closed circuit TV system while everything is being recorded. Another short discussion period follows. I encourage the registrar trainee to be around with the students; although he helps me to look after them I expect him more to learn, through observing me, than to help teach. Thus, each student, by the end of his attachment to child psychiatry, would have been directly involved with one or two cases and indirectly with six or seven others.
The second modality of teaching in the Psychiatry Module is the weekly two-hour child case conference. Here, the two new cases mentioned above are presented by the respective medical students. They are instructed to spend only 15 minutes in their oral presentation but 30 minutes showing relevant excerpts from the videotape; they are also asked to synchronise the two methods of presentation. Usually I require them to present their interview as well as my own. Discussion is again focused on the attitude of the interviewing students and the student-patient relationship, interviewing techniques (theirs as well as mine), psychodynamic formulation, therapeutic strategy in general as well as psychotherapeutic techniques actually demonstrated. Each student attends four such case conferences and has to present in one or two of them. In fact the case conference has also become an important in-service training session for other staff members in the Unit as well as for students of other professional disciplines. The audio-visual system has certainly made our teaching much more tangible, effective and interesting.

The third modality of teaching is the lecture series on child psychiatry. Other than for an introductory lecture in the orientation week, there are six 3-hour afternoon lectures and all students in the module are expected to attend (i.e. not only the students actually doing child psychiatry). In fact this lecture series is more like a series of workshops. The topics covered are:

(a) interviewing the child and the family

(b) development and disorders in development

(c) emotional disorders in childhood

(d) conduct disorders and delinquency

(e) miscellaneous conditions

(f) treatment in child psychiatry.

The topics are chosen so as to emphasise common conditions seen in actual practice and to promote psychosocial awareness in the practice of medicine. Our of the three hours in each lecture, about one to one and a half hours are spent on demonstrating the edited videotapes of actual cases collected over the years. Students are often asked to role-play. I am astounded by how sensible and insightful they are, particularly when they play the part of parents or the presenting patient.

The fourth and last modality of teaching is the weekly psychosocial seminar which takes place not in the Psychiatry Module but in the Paediatrics Module. There are eight of these stretching from the second to the ninth week of the module. Students doing paediatrics are expected to attend all these seminars. They present a paediatric patient to a paediatrician and myself, with discussion centred around psychosocial issues such as the psychological complications of paediatric illness, the impact of the sick child on the family and vice versa. My paediatric colleague and I discuss these sorts of issues with 
the students, thus blending the paediatric and the psychiatric perspectives. We also tackle issues like attitudes of doctors, the doctor-patient relationship, the ward structure and routine, and the skills of talking with children and their parents. Role-play again is often used; for example, about how to talk to a leukaemic child and/or his parents. In the past we permitted students to select cases they wanted to present but recently we have given them eight topics to choose from:

(1) a common non-life-threatening acute illness, e.g. gastroenteritis

(2) a common acute problem with an alarming association: e.g. convulsions

(3) a common, non-life-threatening chronic physical illness, e.g. asthma

(4) a worrying problem in the newborn, e.g. congenital abnormality

(5) a terminal illness, e.g. leukaemia

(6) a chronic long-term physical disorder with a serious prognosis, e.g. beta thalassaemia

(7) a chronic long-term neuro-developmental disorder, e.g. cerebral palsy

(8) a problem of adolescence, e.g. overdose.

This integrated method of teaching is a good example of the philosophy of the medical school which I mentioned earlier. We do it in such a harmonious manner that we serve as good role models for our observing students as to how child psychiatrists and paediatricians can work together fruitfully.

Although child psychiatry is basically a postgraduate, and even to a large extent a post-membership subject, we aim to make it recognisable clinically and academically at the undergraduate level. Probably it is no exaggeration to say that the older generation of doctors in Hong Kong have very little idea about childhood psychiatric morbidity. This results in misdiagnoses and mismanagement of such children. Moreover, the sub-specialty of child psychiatry remains something of a puzzle to these doctors. Hence, we consider conveying to doctors, and even more so to future doctors, the relevance of child psychiatry. This wide visibility of child psychiatry in our undergraduate curriculum is feasible largely because of our philosophy of teaching which, as I have already mentioned, is that our aim is not to turn students into psychiatrists, and even less so, child psychiatrists, but rather to contribute to making them better doctors. But by so doing, we have gained rather than lost. We believe that not only have we achieved our goals to a large extent but we have also managed to attract more young doctors into psychiatry.

\title{
The use of videos to illustrate child psychopathology to medical students
}

\author{
M. E. Garralda, Senior Lecturer and Honorary Consultant in Child and Adolescent \\ Psychiatry, University of Manchester Department of Psychiatry, Booth Hall \\ Children's Hospital, Blackley, Manchester
}

As a relative newcomer to the medical undergraduate curriculum, child psychiatry is still looking for ways in which to best convey its message to students. There is fairly general agreement that this should combine opportunities to assess children or at least to observe the psychiatric assessment of problems and to impart basic information on child development and psychopathology.

A variety of methods are used from traditional didactic lectures to large groups of students, to experiential methods, with role play exercises reenacting childhood, family, medical and therapeutic situations. Discussion groups on communicating with children and systematic training in the use of simple behavioural techniques have also been described (Black, 1985; Cottrell, 1987).
Whatever the methods favoured, there are practical constraints. These include difficulties in obtaining sufficient time within the highly condensed and competitive undergraduate curriculum and the relatively small numbers of child psychiatric teachers. In practice there are wide variations in the amount of time allocated to child psychiatry in different medical schools (Cottrell, 1987).

This report describes an expansion of a child psychiatric teaching programme which was achieved by making use of videotapes. The teaching programme to undergraduates on which this was based and the response by medical students has been described before (Garralda, 1984). The programme takes place during the students' eight week paediatric placement and it consists of introductory lectures in child 